TITLE:

\title{
Fabrication of highly crosslinked methacrylate-based polymer monoliths with well-defined macropores via living radical polymerization
}

\section{$\operatorname{AUTHOR}(S):$}

Hasegawa, George; Kanamori, Kazuyoshi;

Nakanishi, Kazuki; Yamago, Shigeru

\section{CITATION:}

Hasegawa, George ...[et al]. Fabrication of highly crosslinked methacrylate-based polymer monoliths with well-defined macropores via living radical polymerization. Polymer 2011, 52(21): 4644-4647

\section{ISSUE DATE:}

2011-09

URL:

http://hdl.handle.net/2433/148006

\section{RIGHT:}

(c) 2011 Elsevier Ltd.; この論文は出版社版でありません。引用の際には 出版社版をご確認ご利用ください。; This is not the published version. Please cite only the published version. 


\title{
Fabrication of Highly Crosslinked Methacrylate-based Polymer Monoliths with Well-Defined Macropores via Living Radical Polymerization
}

\author{
George HASEGAWA, ${ }^{\mathrm{a}}$ Kazuyoshi KANAMORI, ${ }^{\mathrm{a},{ }^{*}}$ Kazuki NAKANISHI, ${ }^{\mathrm{a}}$ Shigeru YAMAGO ${ }^{\mathrm{b}}$

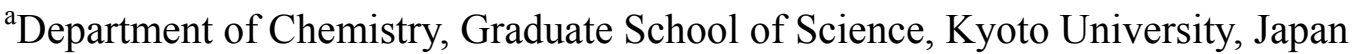 \\ ${ }^{\mathrm{b}}$ Institute for Chemical Research, Kyoto University, Japan
}

\begin{abstract}
*Corresponding author: Kazuyoshi KANAMORI, Dr. Eng.
Department of Chemistry, Graduate School of Science, Kyoto University

Kitashirakawa, Sakyo-ku, Kyoto 606-8502, Japan.

TEL/FAX: +81-75-7 53-7673

E-mail: kanamori@kuchem.kyoto-u.ac.jp
\end{abstract}

\begin{abstract}
Rigid methacrylate-based polymer monoliths with well-defined macropores have been synthesized from glycerol 1,3-dimethacrylate (GDMA) and trimethylolpropane trimethacrylate (Trim) by organotellurium-mediated living radical polymerization. In each system, poly(ethylene oxide) induced spinodal decomposition with the progress of polymerization of GDMA or Trim. Well-defined macroporous structure can be tailored by fixing the bicontinuous structure by the sol-gel transition. Both polymer monoliths possessed macropores with narrow size distributions and the macropore size can be controlled simply by varying the amount of poly(ethylene oxide). Starting from GDMA, polymer monoliths with unimodal macropores can be obtained due to the collapse of micro- and mesopores, which were originally embedded in macropore skeletons, by large shrinkage during drying. In contrast, starting from Trim, the obtained polymer monoliths include not only macropores but also micro- and mesopores, which lead to high specific surface area $\left(470 \mathrm{~m}^{2} \mathrm{~g}^{-1}\right)$, owing to the higher crosslinking density.
\end{abstract}




\section{Keywords}

macroporous methacrylate-based crosslinked monoliths; organotellurium-mediated living radical polymerization; bicontinuous structure

\section{Introduction}

Polymer monoliths have been drawing increasing attention in various fields, especially in separation science. Since the early 1990s, the monolithic separation media (columns) based on rigid polymer networks have been studied as substitutes for the conventional silica-based columns, which cannot be used in aqueous alkaline conditions [1-5]. In particular, polymer monolithic columns are important in the separation of large molecules, such as peptides, proteins and saccharides [6-9]. The polymer monolithic columns were originally prepared in the co-existence of "porogen" (usually poor solvent) by conventional free radical polymerization [10-12]. In such cases, the pores, especially macropores in micrometer sizes, in the polymer monoliths are tailored as the interstices of segregated polymer grains (or "microgels") formed through an abrupt increase of molecular weight. The typical resultant porous structure is aggregation of micron-sized particles with disordered shapes, called "cauliflower-like structure". Hence it accompanies difficulty in the precise control of the pore properties such as pore size and pore volume, though which is indispensable for the development of wide applications polymer monolithic columns. In 2006, we for the first time reported the precise and independent controls of pore size and pore volume in highly crosslinked poly(divinylbenzene) monoliths sorely from divinylbenzene by utilizing living radical polymerization [13], which enables a fine control of polymerization with a gradual increase in molecular weight with reaction time [14]. The well-defined and controlled macropores are tailored by fixing the bicontinuous structure in the appropriate stage of spinodal decomposition $[15,16]$, which can be induced by the addition of another nonreactive polymer, poly(dimethylsiloxane). Since then, there have been several reports regarding the pore control of 
various polymers utilizing various living radical polymerization techniques accompanied by spinodal decomposition [17-21].

In this study, we have fabricated highly crosslinked methacrylate-based polymer monoliths with well-defined macropores from two kinds of methacrylate-based monomers; glycerol 1,3-dimethacrylate (GDMA) and trimethylolpropane trimethacrylate (Trim) by organotellurium-mediated living radical polymerization (TERP) [22,23], which is known as a versatile living radical reaction technique applicable to a wide range of monomers, such as styrenes, acrylates, methacrylates, and acrylamides. The macropores are regulated by polymerization-induced spinodal decomposition in the GDMA/Trim-PEO-solvent pseudo-ternary systems. Variations in pore properties have been investigated in the systems with a fixed concentration of the monomer and a fixed molar ratio of living radical initiator to the monomer.

\section{Experimental}

Glycerol 1,3-dimethacrylate (GDMA) was purchased from Wako Pure Chemical Ind., Ltd. (Japan). The solvent, N,N-dimethylformamide (DMF) was purchased from Kishida Chemical Co., Ltd. (Japan). Trimethylolpropane trimethacrylate (Trim) and poly(ethylene oxide) $\left(M_{\mathrm{W}}=\right.$ 1000000) were obtained from Sigma-Aldrich Co. (USA). The radical initiator, 2,2'-azobis(isobutyronitrile) (AIBN) was purchased from Tokyo Chemical Industry Co., Ltd. (Japan). The mediator for organotellurium living radical polymerization, Ethyl-2-methyl-2-butyltellanylpropionate (BTEE) was kindly donated by Otsuka chemical Co., Ltd. (Japan). All chemicals were used as received without further purification.

Table 1 summarizes the starting compositions studied in our experiments. In the case of poly(glycerol 1,3-dimethacrylate) (PGDMA), since the gelation time was relatively short, the amount of AIBN was fixed as $0.5 \mathrm{~mol} \%$ of GDMA, whereas in the case of poly(trimethylolpropane trimethacrylate) (PTrim), the amount of AIBN was $1 \mathrm{~mol} \%$ of monomer like in the previous reports. The amount of BTEE was fixed as $1 \mathrm{~mol} \%$ of monomer in both cases. A given amount of PEO 
was completely dissolved in the solvent DMF at $60{ }^{\circ} \mathrm{C}$, and then the methacrylate monomer was added. After stirring for $3 \mathrm{~min}$, the resulting homogeneous starting solution was degassed by sonication for $3 \mathrm{~min}$, and then AIBN was added followed by purging with nitrogen supplied using a stainless-steel needle through a silicone resin septum for $10 \mathrm{~min}$. Then, BTEE was added and the resultant solution was mixed at $80{ }^{\circ} \mathrm{C}$ until the reprecipitated PEO was completely dissolved. The solution was subsequently transferred to an ampule and kept at $80{ }^{\circ} \mathrm{C}$ in an oven. After reacted for $24 \mathrm{~h}$, the resulting wet gels were washed with water/ethanol (1/1 in volume) solution to remove the solvent, PEO, and unreacted monomers, etc., followed by a solvent exchange to pure ethanol. Evaporative drying was then conducted at $60{ }^{\circ} \mathrm{C}$ for $24 \mathrm{~h}$. The typical gelation times of PGDMA and PTrim gels were approximately $30 \mathrm{~min}$ and $60 \mathrm{~min}$, respectively. Some PGDMA samples were also dried by supercritical drying (SCD) instead of evaporative drying. In the case of SCD, after washed with ethanol, the samples were subjected to a further solvent exchange with 2-propanol at $60{ }^{\circ} \mathrm{C}$ for $24 \mathrm{~h}$. In the high-pressure autoclave (All-round Smart-operating Isostatic Pressing chamber (ASIP), Mitsubishi Materials, Japan), the wet gels were dried from supercritical carbon dioxide at $80{ }^{\circ} \mathrm{C}$ and $14 \mathrm{MPa}$. Hereafter, samples dried by evaporation are termed as xerogels, and those dried by supercritical drying as aerogels. For the mercury porosimetry and nitrogen sorption described below, the evaporatively-dried samples were thoroughly dried by heating from room temperature to $150{ }^{\circ} \mathrm{C}$ in $90 \mathrm{~min}$ and kept at the temperature for $6 \mathrm{~h}$ in air.

The microstructures of the fractured surfaces of the samples were observed by scanning electron microscopy (SEM) (JSM-6060S, JEOL, Japan). A mercury porosimeter (Pore Master 60-GT, Quantachrome Instruments, USA) was used to characterize the macropores of the samples, while nitrogen adsorption-desorption apparatus (BELSORP-mini II, Bel Japan Inc., Japan) was employed to characterize the meso- and micropores of the samples. Before the nitrogen adsorption-desorption measurement, the sample was degassed at $120{ }^{\circ} \mathrm{C}$ under vacuum for more than $6 \mathrm{~h}$. 


\section{Results and Discussion}

The chemical structures of the methacrylate monomers used in this study are depicted in Figure 1 (a). The monomers GDMA and Trim possess two and three methacrylate groups, respectively, which lead to highly crosslinked rigid polymer networks after polymerization. The crosslinking density in the resultant polymers should be higher in PTrim than in PGDMA. Another difference between these monomers is that GDMA is more hydrophilic than Trim due to the hydroxyl group. Figure 1 (b) shows the appearances of the macroporous PGDMA and PTrim xerogel monoliths which were fabricated from almost the same monomer concentration in volume. Both xerogels kept a crack-free cylindrical shape when the wet gels were carefully dried. The shrinkage during drying in the PGDMA and PTrim monoliths were approximately $29 \%$ and $18 \%$ (in linear), respectively. The larger shrinkage for the PGDMA monoliths was derived from the lower crosslink density. Even when the gels were dried by SCD, shrinkage in approximately $24 \%$ was observed in the case of PGDMA.

Figure 2 shows the macroporous structures of the PGDMA and PTrim monoliths with varying amounts of PEO. It was found that the macroporous morphologies become coarser with increasing amount of PEO in both polymers, suggesting that the phase separation took place between the gel-rich phase consisting of methacrylate-based polymer and the fluidic phase including PEO in both cases. The solvent DMF should distribute in both gel-rich and fluidic phases. However, the amount of PEO to obtain macroporous structure was different in two cases; the PTrim monolith with bicontinuous structure was obtained with $0.04 \mathrm{~g}$ of PEO, while $0.12 \mathrm{~g}$ of PEO was needed to obtain the PGDMA monolith with the similar structure. This fact can be mainly explained by the difference in the compatibility of polymer networks and PEO. The relatively hydrophobic PTrim network exhibits the lower compatibility with hydrophilic PEO. Hence, the small amount of PEO is enough to induce thermodynamic instability (spinodal decomposition). On the other hand, the PGDMA network is more hydrophilic owing to the hydroxyl groups and exhibits the higher compatibility with PEO. Relatively large amount of PEO 
is therefore needed to evoke phase separation in the PGDMA system.

The macropore characteristics of the methacrylate-based polymer monoliths with different amounts of PEO were investigated by mercury porosimetry as shown in Figure 3. It is found that all the monoliths with bicontinuous structure possess narrow pore size distributions. Since the pore properties are governed by spinodal decomposition between polymerizing species and PEO as mentioned above, increasing PEO leads to larger macropores. Because of the larger shrinkage during drying, the macropore volumes of the PGDMA monoliths are about half of those of the PTrim monoliths even when the concentration of monomer (e.g. the solid fraction in the monoliths) is almost the same. The available macropore size of the PGDMA monoliths is smaller compared to the PTrim monoliths for this reason. The change in macropore characteristics with varying the amount of PEO is also different in two systems. In the case of PGDMA, both pore size and pore volume more drastically increase as the increase in the amount of PEO compared to the PTrim system. This is attributed to the fact that the degree of shrinkage during drying decreases with increasing pore size, because the capillary force upon evaporation of solvent from the pores decreases with increasing pore size. On the other hand, such behavior is not evident in the PTrim system owing to the smaller shrinkage. Similar behaviors are also observed in the macroporous metal oxide gels prepared by the sol-gel method accompanied by spinodal decomposition. In the case of silica, the obtained gels are strong enough and shrinkage during drying is limited; however, mechanically weaker alumina and titania also exhibit relatively large shrinkage during the drying, resulting in the similar tendency to the PGDMA system $[25,26]$.

In poly(divinylbenzene) systems using poly(dimethylsiloxane) as the phase separation inducer, secondary phase separation in the separated phases imparts smaller pores with nanometer scales in the macropore walls [19]. The present systems also involve smaller pores as the result of secondary phase separation within the PGDMA/PTrim gel-rich phase. Figure 4 shows the nitrogen adsorption-desorption isotherms of the PGDMA and PTrim xerogels, and the supercritically-dried PGDMA aerogel. Table 2 summarizes the pore properties of each sample. 
As for PGDMA monoliths, although the PGDMA xerogel possesses only the subtle amounts of micro- and mesopores, the SCD-sample possesses the larger amounts of micropores and mesopores; the higher uptake near the $p / p_{0}=0$ and the obvious hysteresis with the increase in adsorption due to the mesopores $\left(p / p_{0} \sim 0.6\right)$ are observed in the isotherm. This result indicates that the micropores and mesopores formed in PGDMA monoliths are collapsed due to the large shrinkage during drying. In contrast, the PTrim xerogel possesses the larger amounts of micropores and mesopores owing to the higher crosslink density. Hence, methacrylate-based polymer monoliths with high specific surface area can be readily obtained in the PTrim system.

\section{Conclusions}

In summary, rigid methacrylate-based polymer monoliths with well-defined macropores have been synthesized by TERP accompanied by spinodal decomposition. Highly crosslinked PTrim monoliths and relatively hydrophilic PGDMA monoliths including hydroxyl groups were synthesized. Because of the lower crosslink density, the PGDMA monoliths showed larger shrinkage during drying, which caused less controllability of macropore size and small surface area. In contrast, the macroporous PTrim monoliths possessed micropores as well as mesopores, which results in higher specific surface area $\left(470 \mathrm{~m}^{2} \mathrm{~g}^{-1}\right)$. It is expected that this synthetic protocol of macroporous methacrylate-based polymer monoliths can be applied to the preparation of separation media for high-performance liquid chromatography (HPLC).

\section{Acknowledgements}

The present work was supported by the Grant-in-Aid for Scientific Research (No. 22.75 for G.H., No. 22750203 for K.K. and 20350094 for K.N.) from the Ministry of Education, Culture, Sports, Science and Technology (MEXT), Japan. Also acknowledged is the Global COE Program "International Center for Integrated Research and Advanced Education in Materials Science” (No. B-09) of the MEXT, Japan, administrated by the Japan Society for the Promotion of Science (JSPS). 


\section{References}

[1] Wang QC, Svec F, Fréchet JMJ. Anal Chem 1993;65(17):2243-2248.

[2] Hoegger D, Freitag R. J Chromatogr A 2001;914(1-2):211-222.

[3] Courtois J, Fischer G, Sellergren B, Irgum K. J Chromatogr A 2006;1109(1):92-99.

[4] Vlakh EG, Tennikova TB. J Sep Sci 2007;30(17):2801-2813.

[5] Buchmeiser MR. Polymer 2007;48(8):2187-2198.

[6] Svec F, Fréchet JMJ. Science 1996;273(5272):205-211.

[7] Krull IS, Sebag A, Stevenson R. J Chromatogr A 2000;887(1-2):137-163.

[8] Zou H, Huang X, Ye M, Luo Q. J Chromatogr A 2002;954(1-2):5-32.

[9] Rohr T, Hilder EF, Donovan JJ, Svec F, Fréchet JMJ. Macromolecules 2003;36(5):1677-1684.

[10] Viklund C, Pontén E, Glad B, Irgum K. Chem Mater 1997;9(2):463-471.

[11] Cooper AI, Holmes AB. Adv Mater 1999;11(15):1270-1274.

[12] Santora BP, Gagné MR. Macromolecules 2001;34(3):658-661.

[13] Kanamori K, Nakanishi K, Hanada T. Adv Mater 2006;18(18):2407-2411.

[14] Braunecker WA, Matyjaszewski K. Prog Polym Sci 2007;32(1):93-146.

[15] Hashimoto T, Itakura M, Hasegawa H. J Chem Phys 1986;85(10):6118-6128.

[16] Nakanishi K. J Porous Mater 1997;4(2):67-112.

[17] Aoki H, Tanaka N, Kubo T, Hosoya K. J Polym Sci: Part A: Polym Chem 2008;46(14):4651-4673.

[18] Kanamori K, Hasegawa J, Nakanishi K, Hanada T. Macromolecules 2008;41(19):7186-7193.

[19] Hasegawa J, Kanamori K, Nakanishi K, Hanada T, Yamago S. Macromolecules 2009;42(4):1270-1277.

[20] Hasegawa J, Kanamori K, Nakanishi K, Hanada T, Yamago S. Macromol Rapid Commun 2009;30(12):986-990.

[21] Zhang R, Qi L, Xin P, Yang G, Chen Y. Polymer 2010;51(8):1703-1708. 
[22] Yamago S, Iida K, Yoshida J. J Am Chem Soc 2002;124(12):2874-2875.

[23] Goto A, Kwak Y, Fukuda T, Yamago S, Iida K, Nakajima M, Yoshida J. J Am Chem Soc 2003;125(29):8720-8721.

[24] Yamago S. Chem Rev 2009;109(11):5051-5068.

[25] Tokudome Y, Fujita K, Nakanishi K, Miura K, Hirao K. Chem Mater 2007;19(14):3393-3398.

[26] Hasegawa G, Kanamori K, Nakanishi K, Hanada T. J Am Ceram Soc 2010;93(10):3110-3115. 


\section{Figures}

(a)<smiles>C=C(C)C(=O)OCC(O)COC(=O)C(=C)C</smiles>

Glycerol 1,3-dimethacrylate (GDMA)<smiles>C=C(C)C(=O)OCC(CC)(COC(=O)C(=C)C)OC(=O)C(=C)C</smiles>

Trimethylolpropanetrimethacrylate (Trim)

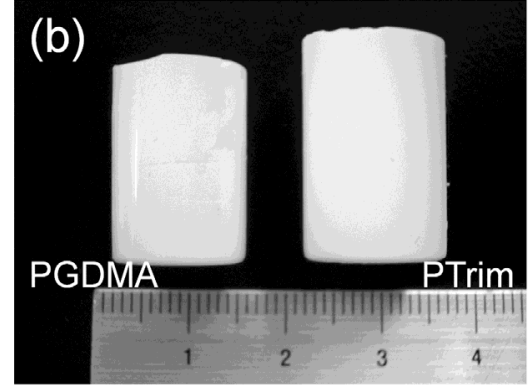

Figure 1 (a) Chemical structures of the methacrylate monomers and (b) appearances of the monolithic PGDMA and PTrim xerogels.
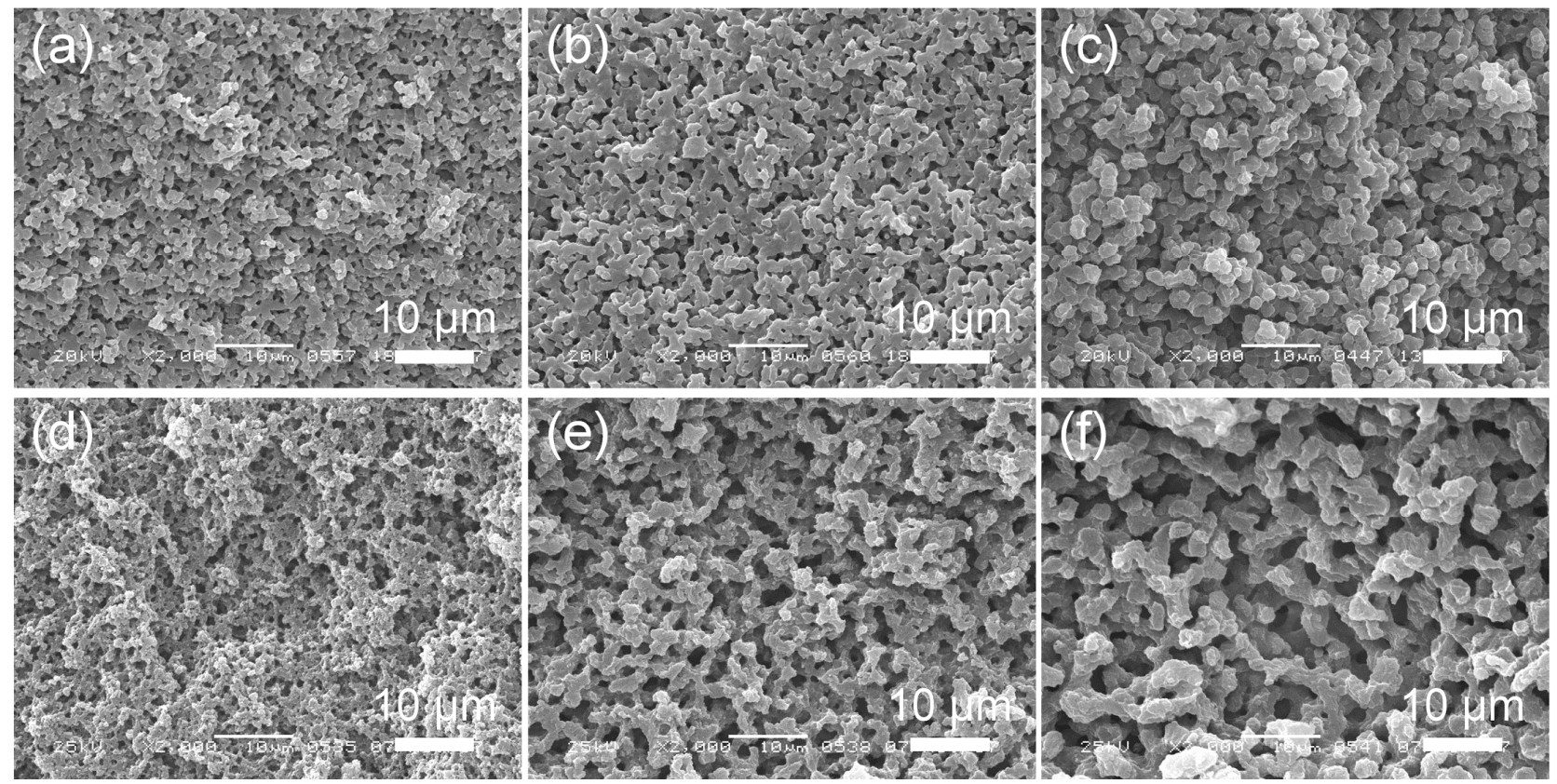

Figure 2 Scanning electron micrographs of the PGDMA xerogels prepared with varied PEO content, (a) G12-0.10, (b) G12-0.12, and (c) G12-0.16, and those of the PTrim xerogels, (d) T12-0.03, (e) T12-0.04, and (f) T12-0.05. 

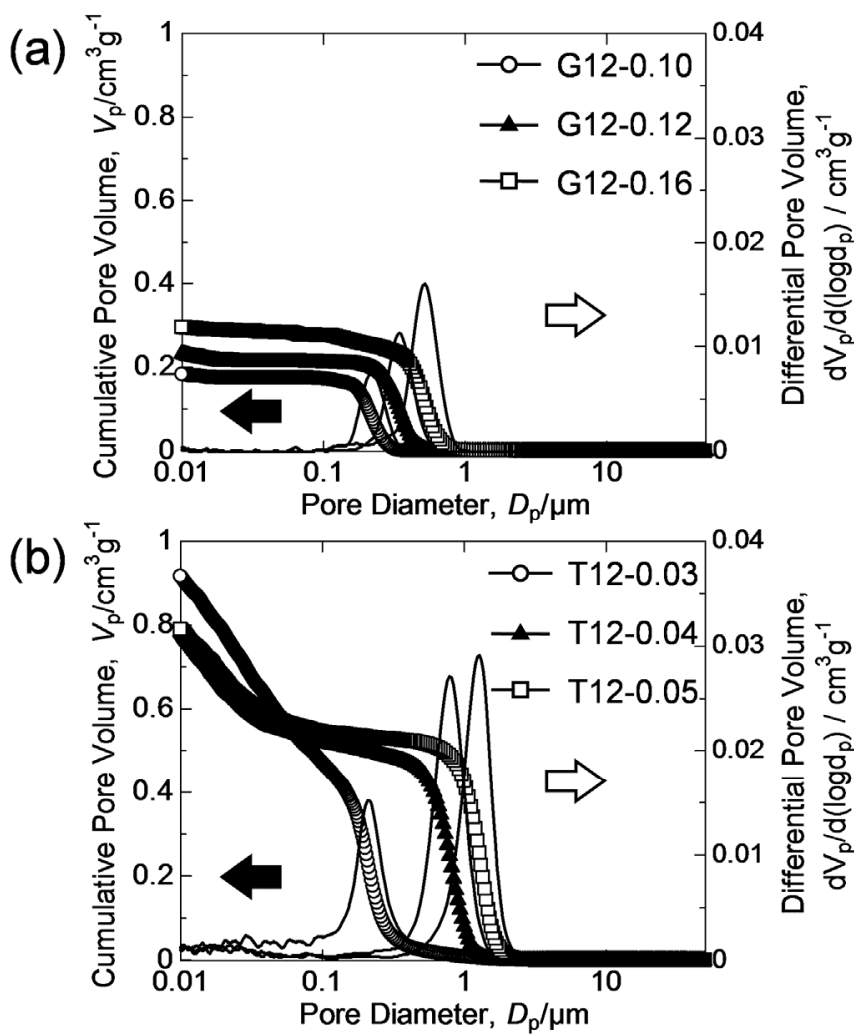

Figure 3 Pore size distributions of the PGDMA and PTrim xerogels prepared with varied amount of PEO.

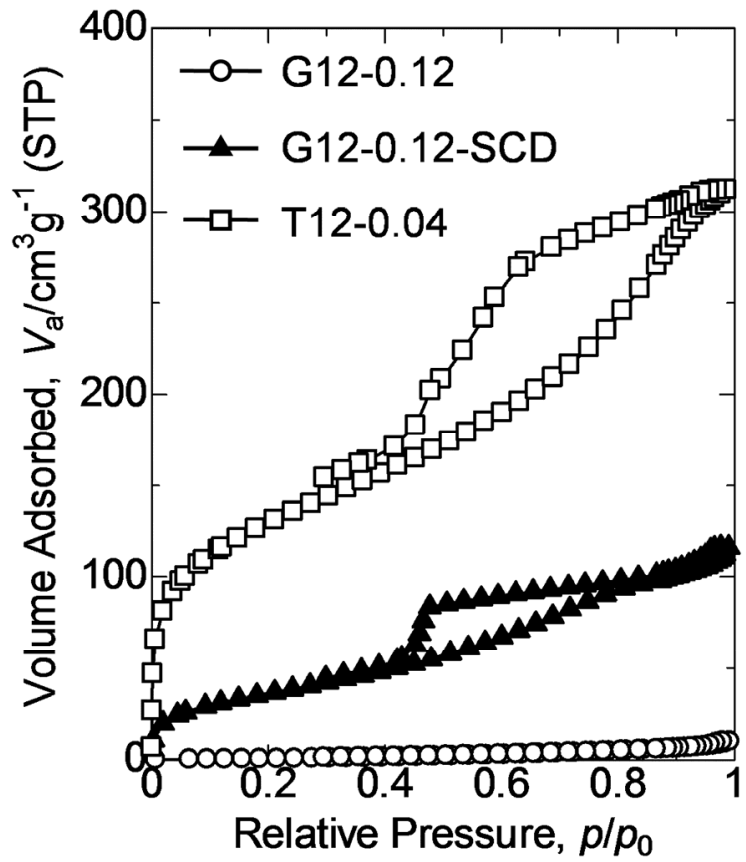

Figure 4 Nitrogen adsorption-desorption isotherms of the PGDMA and PTrim xerogels, and the supercritically-dried PGDMA aerogel. 


\section{Tables}

Table 1 Starting compositions of the samples reacted at $80^{\circ} \mathrm{C}$.

\begin{tabular}{ccccccc}
\hline \multirow{2}{*}{ sample } & GDMA & Trim & DMF & PEO & AIBN & BTEE \\
& $/ \mathrm{mL}$ & $/ \mathrm{mL}$ & $/ \mathrm{mL}$ & $/ \mathrm{g}$ & $/ \mathrm{g}$ & $/ \mu \mathrm{L}$ \\
\hline $\mathrm{G} 12-0.10$ & 5.0 & - & 12 & 0.100 & 0.020 & 56 \\
$\mathrm{G} 12-0.12$ & 5.0 & - & 12 & 0.120 & 0.020 & 56 \\
$\mathrm{G} 12-0.16$ & 5.0 & - & 12 & 0.160 & 0.020 & 56 \\
$\mathrm{~T} 12-0.03$ & - & 5.0 & 12 & 0.030 & 0.026 & 36 \\
$\mathrm{~T} 12-0.04$ & - & 5.0 & 12 & 0.040 & 0.026 & 36 \\
$\mathrm{~T} 12-0.05$ & - & 5.0 & 12 & 0.050 & 0.026 & 36 \\
\hline
\end{tabular}

Table 2 Pore characteristics of the PGDMA and PTrim xerogels, and the supercritically dried PGDMA gels.

\begin{tabular}{|c|c|c|}
\hline sample & $\begin{array}{c}a_{\mathrm{BET}}{ }^{a} \\
/ \mathrm{m}^{2} \mathrm{~g}^{-1}\end{array}$ & $\begin{array}{c}V_{\text {micro-meso }} \\
\quad / \mathrm{cm}^{3} \mathrm{~g}^{-1}\end{array}$ \\
\hline G12-0.12 & 2 & 0.015 \\
\hline G12-0.12-SCD & 130 & 0.180 \\
\hline T12-0.04 & 470 & 0.482 \\
\hline
\end{tabular}

${ }^{a}$ BET specific surface area obtained by nitrogen adsorption.

${ }^{b}$ micro- and mesopore volume obtained from nitrogen absorption isotherms at $p / p_{0}=0.99$. 